\title{
Die Nährstoffgehalte der Miesmuschel (Mytilus edulis) und der Klaffmuschel (Mya arenaria) in ihren jahreszeitlichen Schwankungen
}

\author{
Von Johannes Henschel \\ Aus dem Hygienischen Institut der Hansestadt Hamburg (Direktor Prof. Dr. Dr. Harmsen) \\ und der Biologischen Anstalt Helgoland, List auf Sylt (Direktor Prof. Dr. HAGMEIER) \\ (Mit 9 Tabellen und 6. Abbildungen im Text.)
}

I. Problemstellung.

Die vorliegende Studie behandelt die jahreszeitlichen Schwankungen der Nährstoffgehalte zweier Muschelarten, die als Speisemuscheln Verwendung finden, der Miesmuschel Mytilus edulis und der Klaffmuschel Mya arenaria. Die Untersuchungen wurden im Rahmen einer Zusammenarbeit zwischen dem Hygienischen Institut Hamburg und der Biologischen Anstalt Helgoland*), jetzt List auf Sylt, einige Untersuchungen auch im Institut für Küsten- und Binnenfischerei Hamburg-Cuxhaven ausgeführt. Sie sollten den Ernährungszustand der Muscheln in den verschiedenen Jahreszeiten und aus verschiedenen Zonen des schleswig-holsteinischen Wattenmeeres prüfen, um die ernährungsphysiologischen Grundlagen und damit auch die hygienischen Verhältnisse der besten Fangpläte dieser Tiere zu ermitteln. Mittlerweile ist das Ernährungsproblem in Deutschland nicht mehr so dringlich und das Interesse an diesen Arbeiten daher bis zu einem gewissen Grade erlahmt. Trotgdem wird der Genuß von Speisemuscheln auch weiterhin seine beschränkte Bedeutung behalten, und es erscheint daher berechtigt, vorliegende Ergebnisse auch heute noch $\mathrm{zu}$ veröffentlichen.

In der Literatur liegen für Mytilus wie für Mya Nährstoffuntersuchungen bereits vor. Ich erwähne die alten Tabellen von Buddenberg (siehe KöNIG 1919). Für die hier zur Diskussion stehenden Probleme wichtiger sind die Arbeiten von DeLfF (1912). und Daniel (1921), da auch diese Forscher ihre Untersuchungen jahreszeitlich durchgeführt haben. DeLfFs Miesmuscheln stammten zum größten Teil aus der Kieler Bucht. Bei den Daniel'schen Tieren handelt es sich um englische Muscheln, so daß hier recht verschiedenartiges Material vorlag, was zu interessanten Vergleichen Anlaß geben kann. Für die Auster hat Mrtchell (1916) und Russel (1923) ähnliche Versuche ausgeführt. Aus neuerer Zeit lag eine Arbeit von Rinke (1937) vor.

*) Den Herren der Biologischen Anstalt Helgoland möchte ich für die freundliche Anregung und Unterstützung meiner Arbeit bestens danken, besonders dem Leiter der Anstalt, Herrn Professor Dr. HAGMEIER und Herrn Dr. B. WERNER. 


\section{Materialund Methoden}

Das Material stammt zum größten Teil aus Sylt, zum kleineren Teil aus den Watten bei Friedrichskoog. Die Muscheln wurden an den betreffenden Orten möglichst von den gleichen Stellen gesammelt. Von der Biologischen Anstalt Helgoland wurde auf Sylt für Mytilus eine Muschelbank des im Lister Königshafen gelegenen Watts benutzt, die in den Protokollen als "Äußere Muschelbank“ bezeichnet wird. Die Miesmuscheln des Jahres 1946 allerdings wurden zum größten Teil an der Austernbrücke am Ellenbogen, der Anlegebrücke der Biologischen Anstalt, gesammelt. Die Fangplätze für Mya lagen auch im Watt des Königshafens, der Hauptfangplatz am inneren Rand der als "Außere Muschelbank“ bezeichneten Zone. Ein zweiter Fangplatz lag am Uthörn Sand, am Rande einer Sandbank, deren tiefer gelegene Randzonen nur bei besonders tiefer Ebbe trocken fielen (siehe Karte bei KORNMANN, dieses Heft Seite 56). Über hydrographische und biologische Eigentümlichkeiten dieser Muschelbänke wird von anderer Seite berichtet werden.

Vom Friedrichskoog wurde uns nur Mya geschickt. Die dortigen Muschelbänke, die vom Institut für Küsten- und Binnenfischerei bearbeitet wurden, lagen auf dem "Haken-Sand“ und an der "Peter Kleist-Ecke". Auch hierüber wird noch an anderer Stelle berichtet werden.

Mytilus, die auf ihren Bänken oberflächlich am Boden liegt, kann zur Ebbezeit gesammelt werden, $M y a$, die tief im Schlick versenkt lebt, muß gegraben werden. (Uber Biologie und Fangmethoden von Muscheln im allgemeinen und Mytilus im besonderen siehe HAGMEIER 1916, HAGMEIER und KANDLER 1937, LINKE 1939, P. F. MEYER 1947).

Die Muscheln wurden einmal im Monat gesammelt, möglichst, wenn Wetter und Tide es zuließen, um die Monatsmitte. Die zur Untersuchung ausgewählten Tiere sollten möglichst gleiche Größe haben. Die Schalenlänge von Mytilus schwankte etwa von $5-7 \mathrm{~cm}$. Die Schwankungen waren für $M y a$ größer, die Werte der Schalenlängen lagen zwischen 5 und $11 \mathrm{~cm}$. Mya wird wesentlich größer als Mytilus. Ihre Längenvariation besitzt daher einen viel brẹiteren Spielraum. Jedenfalls hatten alle Muscheln die Geschlechtsre if e erreicht, so daß in dieser Beziehung nur physiologisch gleichwertiges Material zur Untersuchung gelangte.

Nach dem Sammeln wurden die Tiere etwa einen halben bis einen Tag lang in reines Seewasser gelegt, um sich selbsttätig vom Sande zu reinigen. Dann wurden sie gewogen, der Weichkörper aus den Schalen gelöst und in 10prozentigem Formol bzw. in abs. Alkohol fixiert (die letzte Fixierung für die Glykogenbestimmung). Das fixierte Material wurde dem Hygienischen Institut zur weiteren Verarbeitung zugeschickt. Diese ging im Laboratorium folgendermaßen vor sich: Formolmuscheln wurden 2 Stunden, Alkoholmuscheln kurz gewässert, darauf in kleine Würfel geschnitten, bei $85^{\circ} \mathrm{C}$ vorgetrocknet, bei $105^{\circ} \mathrm{C}$ getrodknet, gemörsert und noch einmal getrocknet bis zur Gewichtskonstanz. Die Trockensubstanz wurde nun für die verschiedenen Untersuchungen aufgeteilt.

\section{Die chemischen Bestimmungen.}

Die Trockensubstanz wurde in einer Platinschale gewogen und im Muffelofen bei $500^{\circ} \mathrm{C}$ ver a scht. Eiweiß wurde als Rohprote in nach dem $\mathrm{K}$ jeldalverfahren bestimmt. Im Soxlethapparat wurde das A therlösliche bestimmt, das alle Neutralfette, Phosphatide und Sterine enthält. Die Kohlehydrate wurden als Glykogen bestimmt, bei den Muscheln des Jahres 1946 nach der Methode von Spaeth und Grohmann (Beythien 1944), nach welcher die Substanz in 60prozentiger Kalilauge bis zur Zerstörung gekocht und das Glykogen mit 96prozentigem Alkohol gefällt wird. Nach Waschen mit 66prozentigem Alkohol wird das Glykogen in 2,2prozentiger Salzsäure 3 Stunden invertiert und der Traubenzucker nach FenLING bestimmt. Der Zuckerwert multipliziert mit 0,927 ergibt den Glykogenwert. 1947 begnügte ich mich mit sehr kleinen Mengen Trockensubstanz von nur etwa 0,5-0,1 g Untersuchungsmaterial, das sofort in 2,2prozentiger $\mathrm{HCl}$ gekocht wurde. Die Eiweißstoffe der Zuckerlösung wurden im neutralen Milieu mit $2 \mathrm{ccm} Z$ inksulfat und $2 \mathrm{ccm}$ Ferrocyankali gefällt, der im Filtrat befindliche Traubenzucker nach FeHLiNG bestimmt und darnach der Glykogenwert berechnet. Die Methode ergab befriedigende Resultate und erfaßte auch die Kohlehydrate mit, die möglicher- 
weise im Muschelkörper als Monohydrate vorhanden sind (Siehe RINkE 1937-39).

Die Phosphatide wurden aus 96prozentigem Alkohol gewonnen. Die Phosphorsäure dieser Stoffgruppe wurde nach Juckenack (Beythien 1944) als Phosphormolybdat bestimmt und der Gehalt der Phosphatide aus der Phosphorsäuremenge als Dioleolecithin berechnet.

\section{Die Ergebniss e.}

a) Allgemein.

Als Beispiel des Versuchsganges sei zunächst je eine Untersuchungsreihe von Mytilus und Mya gegeben (Tabelle 1).

Mytilus edulis. Fangplat5: List, Königshafen, Äußere Muschelbank.

Datum: 18. 9. 47. Die Zahlen in Klammern geben die Zahlen der untersuchten Tiere an.

\begin{tabular}{|c|c|c|c|c|c|}
\hline $\begin{array}{l}\text { Wassergeh. in \% } \\
\text { des Fleischgew. }\end{array}$ & $\begin{array}{l}\text { Rohprotein } \\
\text { in } \% \text { Gesam }\end{array}$ & $\begin{array}{l}\text { Atherextrakt } \\
\text { t trodkengew. }\end{array}$ & Glykogen & Asche & Phosphatid \\
\hline $\begin{array}{l}85,2(2) \\
73,5\end{array}$ & $\begin{array}{l}59,8(2) \\
60,2(3)\end{array}$ & $\begin{array}{r}12,2(2) \\
8,8(3)\end{array}$ & $\begin{array}{l}22,1(1) \\
25,8(1) \\
21,7(1) \\
22,6(1) \\
24,4(1) \\
24,0(1)\end{array}$ & $5,9(6)$ & $1.9(5)$ \\
\hline 79,4 & $\begin{array}{l}60,0 \\
\text { Mya arenaria. }\end{array}$ & $\begin{array}{c}10,5 \\
\text { Fangplatz, } \%\end{array}$ & $\begin{array}{l}23,4 \\
\text { Len, Datu }\end{array}$ & $\begin{array}{l}\mathbf{5 , 9} \\
\text { Mytilus. }\end{array}$ & 1,9 \\
\hline $\begin{array}{l}70,9(3) \\
75,6(3)\end{array}$ & $\begin{array}{l}61,5 \text { (I) } \\
73,4 \text { (1) } \\
65,5 \text { (1) } \\
66,8\end{array}$ & $\begin{array}{r}10,3(1) \\
7,7(1)\end{array}$ & $\begin{array}{l}14,6(1) \\
10,6(1) \\
18,6(1) \\
14,6\end{array}$ & $7,5(3)$ & $\begin{array}{l}3,6(1) \\
4,2(1)\end{array}$ \\
\hline
\end{tabular}

Tabelle 1.

Das Gesamtergebnis der Untersuchungen des Jahres 1947 bringt eine weitere Tabelle:

\begin{tabular}{lrlllllll} 
& $\begin{array}{c}\text { Zahl der unter- } \\
\text { suchten Tiere }\end{array}$ & Wasser & Rohprotein & Atherextr. & Glykogen & Asche & Phosphatid \\
\hline Mytilus & 146 & 80,9 & 12,1 & 1,7 & 3,8 & 1,2 & 0,7 \\
Mya & 72 & 79,9 & 13,8 & 1,9 & 2,4 & 1,7 & 0,7
\end{tabular}

Tabelle 2.

Chemische Zusammensetzung von Mytilus und Mya aus dem Versuchsjahr 1947/48. Herkunft der Muscheln List auf Sylt.

Zahlen: Prozentzahlen bezogen auf das Fle is ch gewicht der fixierten Tiere.

Der Wassergehalt von ungefähr $80 \%$ wird nicht ganz dem der frischen Muscheln entsprechen, sondern etwas zu niedrig sein. Die Wasserwerte von KöNIG liegen für die Miesmuschel mit $86,74 \%$, für die Klaffmuschel mit $83,46 \%$ auch wirklich höher. Nur gering dagegen ist der Unterschied mit den Werten von DanieL. Der Jahresdurchschnitt des Wassergehalts seiner Miesmuscheln beträgt $81,7 \%$. Im allgemeinen ist die Zusammensetzung der Muschelkörper beider Arten recht ähnlich. Mytilus enthält mehr Glykogen als 
Mya, dementsprechend liegt der Rohproteinwert für Mya etwas höher. Die Werte des Atherlöslichen gleichen sich fast ganz, die Phosphatidwerte vollständig. Mya hat einen etwas höheren Aschegehalt, was auf einen stets etwas höheren Sandgehalt zurückzuführen ist. Im Gegensatz zu dem Gesamtdurchschnitt zeigen aber die Monatsmittel der beiden Arten erhebliche Unterschiede, die weiter unten noch genau analysiert werden müssen.

b) Mytilus edulis.

Die Ergebnisse sind tabellarisch zusammengefaßt (Tab. 3) und in Kurven (Abb. 1) dargestellt. In dem Verlauf der Kurven spiegeln sich natürlich Vorgänge sehr verschiedener Art wieder. Es können einmal Verschiebungen der

\begin{tabular}{|c|c|c|c|c|c|c|c|}
\hline \multicolumn{2}{|c|}{ Monat } & \multirow{2}{*}{$\frac{\text { Protein }}{69,0}$} & \multirow{2}{*}{$\frac{\text { Fett }}{8,2}$} & \multirow{2}{*}{$\frac{\text { Glykogen }}{22,8}$} & \multirow{2}{*}{ Phosphatid } & \multirow{2}{*}{$\begin{array}{c}\begin{array}{c}\text { Index: } \\
\text { Fleisch- } \\
\text { Schalengew. }\end{array} \\
0,53\end{array}$} & \multirow{2}{*}{$\frac{\begin{array}{c}\text { Zahl der } \\
\text { Tiere }\end{array}}{6}$} \\
\hline III & 1947 & & & & & & \\
\hline IV & 1947 & 73,5 & 11,5 & 15,1 & - & 0,69 & 6 \\
\hline $\mathrm{V}$ & 1947 & 65,8 & 17,2 & 17,0 & - & - & 6 \\
\hline VI & 1.947 & 66,3 & 14,8 & 19,0 & 7,7 & 0,54 & 14 \\
\hline VII & 1947 & 53,3 & 9,3 & 27,4 & 2,7 & 0,53 & 16 \\
\hline VIII & 1947 & 67,3 & 7,1 & 25,7 & 2,6 & 0,40 & 16 \\
\hline IX & 1947 & 63,3 & 11,5 & 25,4 & 2,0 & 0,51 & 16 \\
\hline$X$ & 1947 & 62,2 & 9,0 & 28,8 & 2,0 & 0,68 & 12 \\
\hline XI & 1947 & 66.2 & 8,7 & 25,2 & 2,3 & 0,57 & 20 \\
\hline$I$ & 1948 & 75,0 & 8,7 & 16,3 & 3,5 & 0,54 & 36 \\
\hline II & 1948 & 79,7 & 8,5 & 11,9 & 4,5 & 0,58 & 20 \\
\hline
\end{tabular}

Tabelle 3

(s. Abb. 1)

Monatliche Nährstotfgehalte von Mytilus edulis in \% des aschefreien Trockengewichts während der Untersuchungsperiode 1947/48.

verschiedenen organischen Substanzen, Verschiebungen zwischen Glykogen, Fett und Eiweiß eingetreten sein, wobei die Gesamtmenge des Organischen gleichblieb, etwa dadurch, daß im Betriebsstoffwechsel Anderungen auftraten. Es kann aber auch ein Aufbau oder Abbau, wie es im Bau- oder Hungerstoffwechsel eintritt, stattgefunden haben, wobei die absolute Menge des Organischen zu- oder abnehmen muß. Ein Fixum, woran die absoluten Veränderungen gemessen werden können, besitzen wir nicht, da alle Größen veränderlich sind, die einzelne organische Substanz, Gesamtfleischgewicht, das Schalengewicht und die Größe. Immer aber werden die Maße, die die Schalen betreffen, sich am langsamsten verändern. Auch ist das Schalenwachstum nur auf eine relativ kurze Jahreszeit beschränkt (Havinga 1929). Daher erscheint es berechtigt, die relativ sehr veränderlichen Werte der organischen Substanz zum Schalengewicht in Beziehung zu setzen. Es wurde daher für die einzelnen Monate ein Index ausgerechnet, der das Verhältnis vom Fleisch- zum Schalengewicht ausdrückt. Das Fleischgewicht von Mytilus ist immer kleiner als das Schalengewicht, der Index ist also ein echter Bruch. Ein Vergleich der Indexkurve, die nach diesen Werten gezeichnet wurde, mit dem Verlauf der einzelnen Nährstoffkurven kann nun einen recht interessanten Aufschluß über die Stoffwechselvorgänge geben, die stattgefunden haben, wenn man das herbstliche Schalenwachstum der Muschel gleichzeitig in Rechnung stellt. 
Der Anstieg der Kurve des Ätherextrakts hängt zweifellos mit dem Aufbau der Geschlechtsprodukte zusammen, die besonders reich an solchen Stoffen sind. Das Maximum bezeichnet den Beginn, der abfallende Schenkel die Abgabe der Laichprodukte, also den Höhepunkt der Laichzeit. Hiermit harmoniert der Verlauf der Indexkurve, die zunächst ansteigt, später ebenfalls sinkt. Es tritt durch die Laichabgabe ein Gewichtsverlust des gesamten Tieres ein.
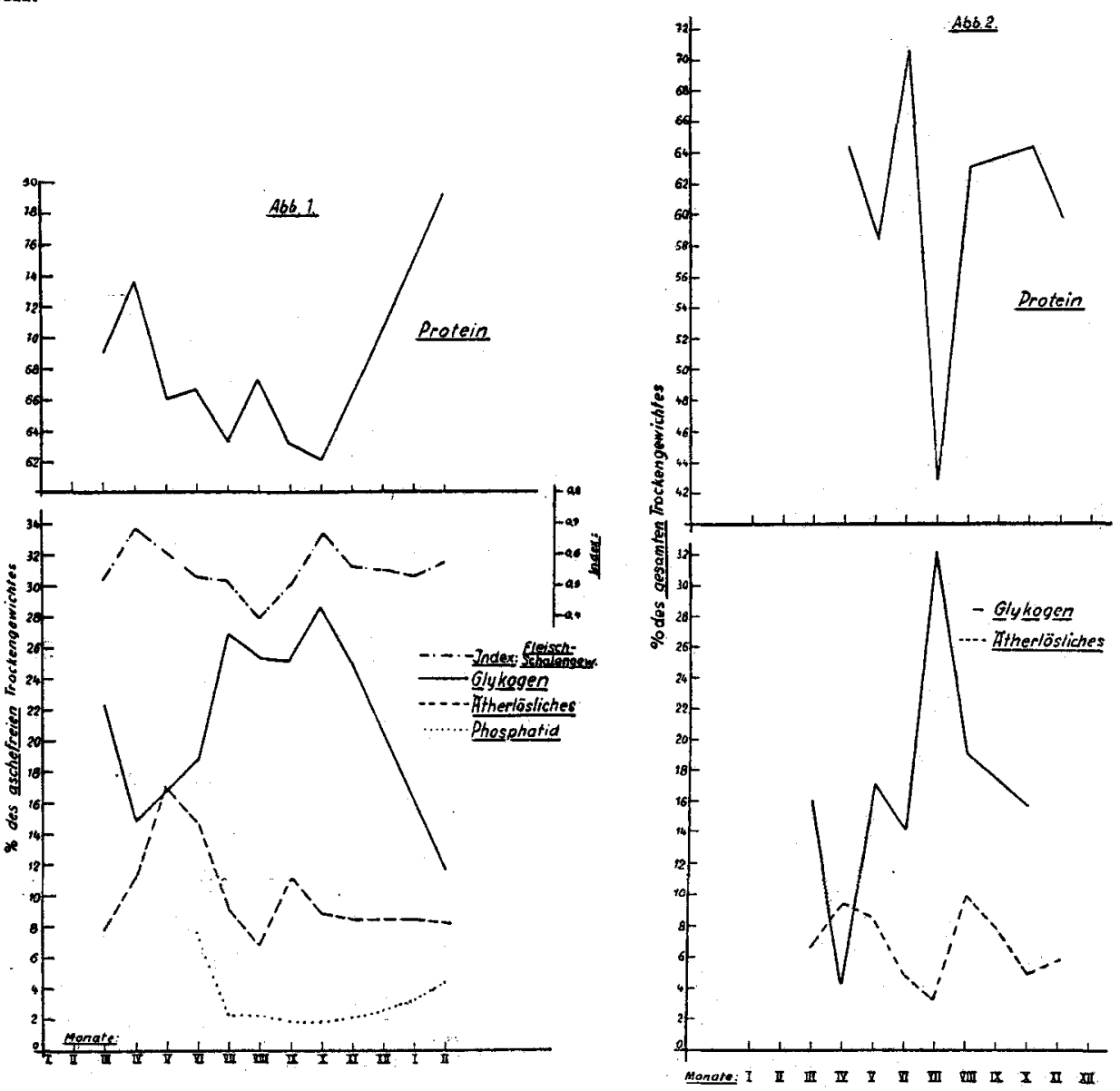

Abb. 1. Monatliche Nährstoffgehalte von Mytilus edulis in $\%$ des aschefreien Trockengewichts während der Untersuchungsperiode 1947/48.

Abb. 2. Monatliche Nährstoffgehalte von Mytilus edulis in $\%$ des gesamten Trockengewichts während der Untersuchungsperiode 1946.

Wir haben einen zweiten Nebengipfel im September, der wohl keine Beziehung zur Laichzeit hat. Es handelt sich wahrscheinlich um eine Anreicherung von Fettsubstanz im Muschelkörper, bedingt etwa durch ölreiche Planktonnahrung, wie wir vielfach bei Planktonfressern Beziehungen zwischen Fettgehalt und aufgenommener Planktonnahrung finden(WIMPENEY 1938). Für diese Auffassung spricht der Verlauf der Phosphatidkurve. Die Phosphatide wurden erst vom Juni des betreffenden Jahres an untersucht. Die Phosphatidkurve zeigt einen zum Atherextrakt parallel laufenden Abfall. In den Laichprodukten spielt das Phosphatid eine besondere Rolle und der Gehalt an 
diesem Stoff muß sich daher durch die Abgabe des Laichs im Kurvenverlauf widerspiegeln. Bei dem zweiten Gipfel der Ätherextraktkurve wurde kein Aufsteigen "und Absteigen des Phosphatidgehaltes beobachtet. Ein Hinweis darauf, daß es sich hier lediglich um eine Anreicherung von Neutralfett gehandelt hat.

Anstieg und Abfall der Proteinkurven im Frühjahr gehen mit der Atherextraktkurve parallel. In den Laichprodukten werden natürlich auch Eiweißstoffe aufgestapelt, die mit dem Ablaichen verloren gehen. Aber diese Eiweißverluste können natürlich im Kurvenverlauf nicht so auffällig zum Ausdruck kommen, wie die Verschiebungen der fettlöslichen Substanzen, da die Eiweißstoffe der Laichprodukte einen viel geringeren Prozentsaz des Gesamteiweißes ausmachen, als es die ätherlöslichen Stoffe in bezug auf die Gresamtkörperfette tun, an denen die Muschel relativ arm ist. Auf der anderen Seite steht die Schwankung des Proteingehaltes natürlich auch in Korrelation zur Glykogenschwankung. Eine Abnahme des Glykogengehaltes muß notwendigerweise eine Zunahme des relativen Eiweißgehaltes nach sich ziehen. Die Glykogenkurve nun fällt im Frühjahr. Es trat trot einer Zunahme des Gesamtgewichtes, ersichtlich aus der Indexkurve, ein Glykogenverlust ein. Ein Teil des Vorrats wird zu dieser Zeit wohl in Fett umgewandelt, das den Laichprodukten zugute kommt. Nach BRUCE (1926) liegt der respiratorische Quotient in den Wintermonaten bis ins Frühjahr hinein höher als 1, was auf eine Umwandlung von Kohlehydraten in Fette durch den inneren Stoffwechsel der Tiere hinweist. Diese Korrelation Glykogen-Fett ist indessen nicht genau. Der Tiefpunkt des Glykogens liegt bereits im April, während die Kulmination der Fettkurve erst in den Mai fällt. Auch bei der Betrachtung weiterer Kurven wird man nicht immer genaue Korrelationen finden, es können noch andere Faktoren die Höhe des Glykogenreichtums, seine Zu- und Abnahme bestimmen.

Nach der Laichabgabe setzt die Periode des vegetativen Wachstums ein. Zunächst werden die Glykogenspeicher aufgefüllt, kurvenmäßig zum Ausdruck gebracht durch einen Anstieg der Glykogenkurve, später einen leichten Anstieg der Indexkurve. Es zeigt sich an der Proteinkurve, daß die Eiweißzunahme zunächst nur gering gewesen sein konnte, da der relative Proteingehalt zunächst noch ein wenig abnimmt. Erst im Herbst setzt ein Vorgang ein, den ich als herbstliche Glykogen-Protein-Verschiebung bezeichne. Einer Abnahme des relativen Glykogengehaltes steht eine Zunahme des relativen Proteingehaltes gegenüber. In dieser Periode werden offenbar die neuen Eiweißbausteine zum Gesamtwachstum des Tieres angebaut. In diese Zeit fällt auch das Schalenwachstum (HAviNGA). Das Fleischgewicht nimmt relativ $a b$, die Indexkurve muß notwendigerweise fallen.

Die besprochenen jahreszeitlichen Schwankungen der Nährstoffgehalte beziehen sich nur auf ein einzelnes Jahr, das natürlich unter ganz bestimmten hydrographischen Bedingungen gestanden hat. Wie wirken sich nun die klimatischen Schwankungen auf den Jahreszyklus dieser Tiere aus? Hierauf kann uns eine weitere Untersuchungsreihe aus dem Jahre 1946 (siehe Tab. 4 und Abb. 2) sowie zwei Arbeiten, die in anderen Gegenden und anderen Jahren ausgeführt wurden, Auskunft geben. Es handelt sich bei den Arbeiten um die schon zitierten Untersuchungen von DeifF aus den Jahren 1901, 1910 und 1911 (Tab. 5 und Abb. 3) und um die Daniel'sche Arbeit aus dem Jahre 1921 (Tab. 6 und Abb. 4). 


\begin{tabular}{lcccc}
\hline Monat & Protein & Fett & Glykogen & Zahl der Tiere \\
\hline III & - & 6,6 & 16,1 & 7 \\
IV & 64,3 & 9,3 & 4,1 & 7 \\
V & 58,4 & 8,7 & 17,3 & 3 \\
VI & 70,3 & 5,1 & 14,2 & 5 \\
VII & 42,6 & 3,2 & 32,2 & 6 \\
VII & 63,3 & 9,9 & 19,4 & 5 \\
X & 64,4 & 5,0 & 15,8 & 7 \\
XI & 59,8 & 6,0 & - & 2
\end{tabular}

Tabelle 4 .

(s. Abb. 2)

Monatliche Nährstoffgehalte von Mytilus edulis in \% des gesamten Trockengewichts während der Untersuchungsperiode 1946.

\begin{tabular}{lcrc} 
Monat & Protein & Fett & Glykogen \\
\hline I & 60,6 & 9,1 & 29,5 \\
IV & 69,8 & 18,2 & 12,2 \\
VII & 56,7 & 8,9 & 34,4 \\
X & 62,8 & 6,3 & 30,5 \\
XII & 56,8 & 11,5 & 31,8
\end{tabular}

Tabelle 5.

(s. Abb. 3)

Monatliche Nährstoffgehalte von Mytilus edulis in \% des aschefreien Trockengewichts, berechnet nach den Untersuchungen von DELFF.

\begin{tabular}{lcrc} 
Monat & Protein & Fett & Glykogen \\
\hline I & 65,7 & 7,4 & 25,2 \\
II & 66,1 & 14,4 & 19,6 \\
III & 76,6 & 9,1 & 14,3 \\
IV & 71,1 & 11,1 & 17,7 \\
V & 66,3 & 4,7 & 29,1 \\
VI & 61,7 & 6,3 & 32,1 \\
VII & 60,7 & 5,6 & 33,8 \\
VIII & 58,6 & 7,9 & 33,6 \\
IX & 55,8 & 7,0 & 37,3 \\
X & 57,3 & 6,6 & 36,1 \\
XI & 60,1 & 8,1 & 31,7 \\
XII & 59,3 & 8,1 & 32,7
\end{tabular}

Tabelle 6 .

(s. Abb. 4)

Monatliche Nährstoffgehalte von Mytilus edulis in \% des aschefreien Trodkengewichts. berechnet nach den Untersuchungen von DANIEL.

Die absoluten Werte der verschiedenen Untersuchungen sind nicht miteinander vergleichbar, Delfrs Bestimmungsmethoden waren andere als die Daniels und auch die der hier vorliegenden Arbeit. Bei meinen Serien des Jahres 1946 konnte ich die Prozentwerte nicht auf das aschefreie Trockengewicht beziehen, da nicht in allen Fällen Aschebestimmungen durchgeführt wurden. Auch diese Werte sind daher mit denen der 1947er Muscheln nicht direkt vergleichbar. Dagegen können wir die gesetgmäßigen Schwankungen, wie sie in allen Kurvenabbildungen zum Ausdruck kommen, sehr wohl miteinander vergleichen. Die Beobachtungen des Jahres 1947 zeigen die Einflüsse eines extrem kalten Winters, des Winters 1946/47, nach welchem die Laichperiode stark verzögert (Mai bis Juli) auftrat, während sie in normalen Jahren, etwa bei Der.frs Versuchen und im Jahre 1946, im April liegt. Bei 
DaniEls englischen Muscheln ist sie noch weiter nach rückwärts verschoben in den Monat Februar. Die Glykogenkurve steigt 1947 bereits vor der Laichzeit an (s. S. 92). Während bei den DelfF'schen Tieren Fettmaximum und Glykogenminimum sich entsprechen, liegt der Glykogenanstieg bei den Daniel'schen Muscheln erst hinter dem Fettgipfel. Auch hierin-werden sich klimatische Einflüsse geltend machen. Wenn nämlich die Laichzeit sich stark verzögert, kann bei ihrem Beginn die frühjahrsmäßige Planktonproduktion so weit gestiegen sein, daß die zur Verfügung stehenden Nahrungsmengen bereits eine Wiederauffüllung der Glykogenspeicher gestatten, obwohl noch immer Laichprodukte gebildet werden und daher Glykogen noch immer zu Fett umgebaut wird. Gerade an den deutschen Küsten wird dieser Fall häufiger eintreten. Nach einer Vereisung des Wattenmeeres pflegt hier mit einer ausgeprägten Westwindwetterlage frühjahrsmäßige Wärme einzusetzen. Mit dieser Westwindwetterlage werden aber auch die in anderen wärmeren Gebieten schon produzierten Planktonmengen herangetragen und stehen den Muscheln zur Verfügung zu einer Zeit, in der sie jahreszyklusmäßig sehr zurückgeblieben sind. Anders an den englischen Küsten. Hier fällt die Laichzeit in eine sehr frühe Periode, in eine Periode, in der auch das frühjahrsmäßige Plankton noch nicht den Höhepunkt seiner Produktion erreicht hat. Auch der ablaichenden Muschel, wenn sie auch keine neuen Laichprodukte mehr bildet, steht noch nicht genügend Nahrung zur Verfügung, um die Energie für die gesteigerte
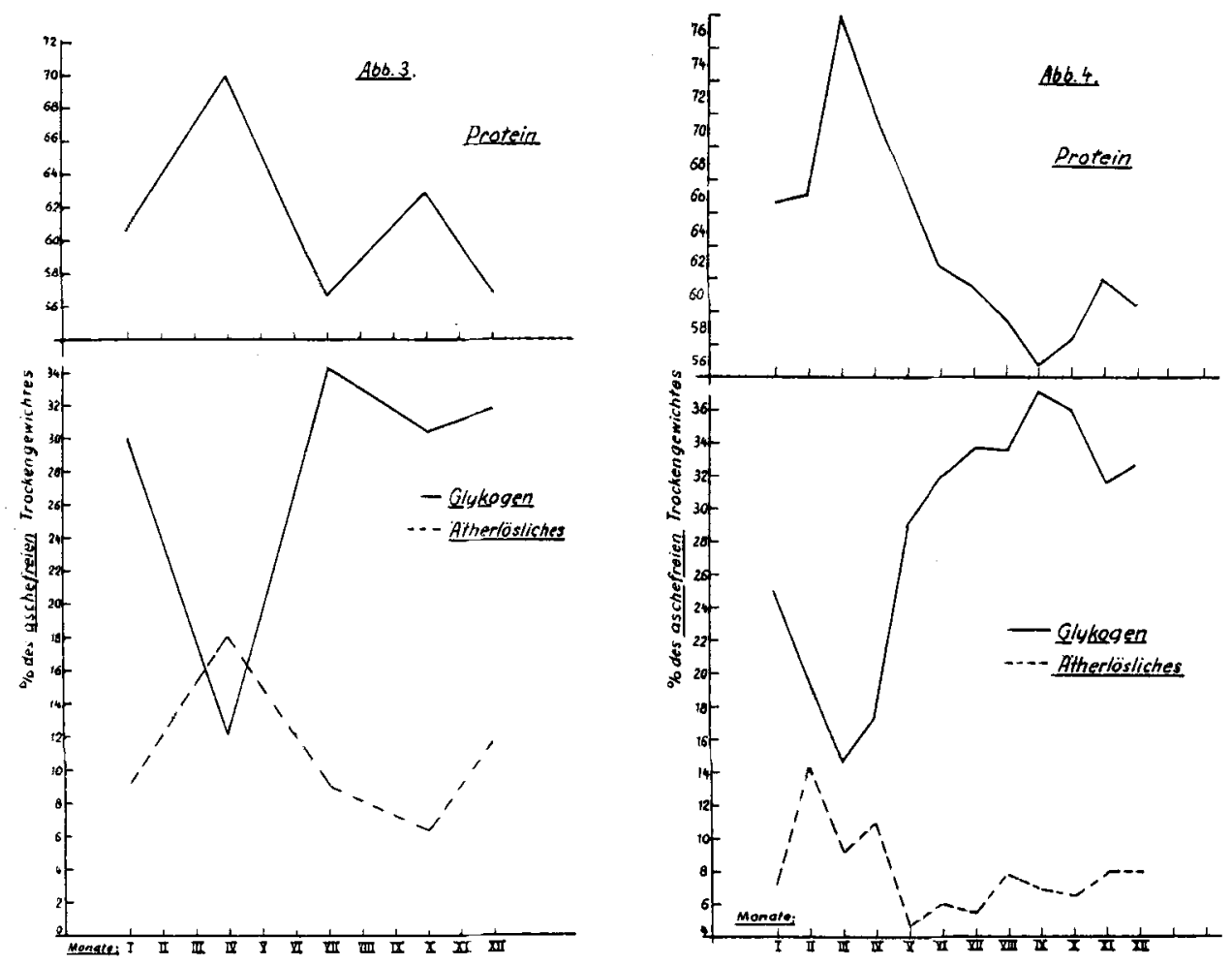

Abb. 3. Monatliche Nährstoffgehalte von Mytilus edulis in $\%$ des aschefreien Trockengewichts, berechnet nach den Untersuchungen von DELFF.

Abb. 4. Monatliche Nährstoffgehalte von Mytilus edulis in \% des aschefreien Trodkengewichts, berechnet nach den Untersuchungen von DANIEL. 
Lebenstätigkeit, die sie ja im Frühjahr auf jeden Fall zeigt, zu decken. Sie muß weiter von den Glykogenvorräten zehren. Das Minimum des Glykogens tritt also verzögert auf.

Auch im weiteren Kurvenverlauf wirken sich offenbar Klimaeinflüsse aus. Bemerkenswert ist der zweite Gipfel der Atherextraktkurve im Jahre 1946. Die Tiere haben in diesem Jahr unter besonders günstigen Bedingungen gestanden, so daß auch in diesem Jahre, wie mir Herr Prof. Hagmeier mündlich bestätigte, sich eine zweite nachhinkende Laichperiode ausbilden konnts. Bemerkenswert ist ferner die Tatsache, daß der Glykogenschwund, der durch die herbstliche Glykogen- und Proteinverschiebung auftritt, in dem ausgesprochen warmen Winter 1947/48 viel stärker war (siehe Februar 1948), als es bei den anderen Muscheln, auch z. B. bei den Muscheln des kalten Winters 1946/47 der Fall war (März 1947). Denn diese sind mit höheren Glykogenvorräten ins Frühjahr 1947 gegangen als die 1947er Tiere ins Frühjahr 1948. Hier bleibt wohl nur die eine Erklärung möglich, daß die Tiere im warmen Winter einen höheren Aktivitätsgrad gezeigt hatten, als es bei den niedrigen Temperaturen des kalten Winters möglich war, und auch im allgemeinen in normalen Wintern der Fall ist. Die Wachstumsvorgänge scheinen nicht zum Abschluß gekommen zu sein, während doch die Nahrungszufuhr immer weniger mit den Bedürfnissen Schritt halten konnte.

Gegenüber den deutschen Küsten sind die Verhältnisse bei den englischen Muscheln ganz allgemein ausgeglichener, die Extreme geringer, wie es auch den mehr ausgeglichenen Lebensraumbedingungen dieser westlichen Gebiete entspricht.

\section{c) Mya arenaria.}

Gegenüber Mytilus zeigt Mya (s. Tab. 7 und 8 und Abb. 5 und 6) eine starke Verschiebung der Laichzeit in die Sommermonate. Dadurch bekommen die Kurven, besonders die Glykogenkurven, ein anderes Bild als es bei Mytilus der Fall ist. Mya speichert weniger Glykogen, geht daher auch mit mehr erschöpften Glykogenvorräten in die frühjahrsmäßige Aktivitätsperiode ein. Es läuft der Laichzeit daher eine längere Glykogenspeicherperiode vorweg, wie es in den Glykogenkurven deutlich zum Ausdruck kommt. Erst im Juni und

\begin{tabular}{|c|c|c|c|c|c|c|c|}
\hline \multicolumn{2}{|c|}{ Monat } & \multirow{2}{*}{$\frac{\text { Protein }}{87,7}$} & \multirow{2}{*}{$\frac{\text { Fett }}{2,9}$} & \multirow{2}{*}{$\frac{\text { Glykogen }}{9,4}$} & \multirow{2}{*}{$\underbrace{\text { Phosphatid }}_{-}$} & \multirow{2}{*}{$\begin{array}{c}\begin{array}{c}\text { Index: } \\
\text { Fleisch- } \\
\text { Schalengew. }\end{array} \\
0,63\end{array}$} & \multirow{2}{*}{$\frac{\begin{array}{c}\text { Zahl der } \\
\text { Tiere }\end{array}}{4}$} \\
\hline III & 1947 & & & & & & \\
\hline IV & 1947 & 71,8 & 12,1 & 16,3 & - & 0,83 & 3 \\
\hline V & 1947 & 72,2 & 10,6 & 17,2 & - & - & 2 \\
\hline VI & 1947 & 67,2 & 14,9 & 17,6 & - & 1,09 & 11 \\
\hline VII & 1947 & 62,4 & 28,3 & 9,2 & 8.6 & 0,71 & 8 \\
\hline VIII & 1947 & 78,2 & 8,0 & 13,9 & 3,8 & 0,65 & 8 \\
\hline IX & 1947 & 74,0 & 9,9 & 16,2 & 4,3 & 0,56 & 9 \\
\hline $\mathrm{X}$ & 1947 & 77,0 & 7,4 & 15,6 & 0.8 & 0,50 & 8 \\
\hline$X I$ & 1947 & 81,6 & 5,0 & 13,4 & 32 & 0,56 & 8 \\
\hline I & 1948 & 83,8 & 7,7 & 8,6 & 3,0 & 0,65 & 18 \\
\hline II & 1948 & 81,8 & 7,5 & 10,5 & 3,9 & 0,71 & 8 \\
\hline
\end{tabular}

Tabelle 7.

(s. Abb. 5)

Monatliche Nährstoffgehalte von Mya arenaria in \% des aschefreien Trockengewichts während der Untersuchungsperiode 1947/48. 


\begin{tabular}{lcccc} 
Monat & Protein & Fett & Glykogen & Zahl der Tiere \\
\hline IV & 64,3 & 3,1 & 5,5 & 3 \\
V & 60,6 & 7,7 & 8,1 & 5 \\
VI & 65,2 & 3,2 & 9,3 & 8 \\
VII & 57,3 & 1,3 & 13,0 & 3 \\
VIII & 66,8 & 3,7 & - & 2 \\
IX & 66,7 & 4,2 & - & 3 \\
X & 63,5 & 4,2 & 8,7 & 5 \\
XI & 58,0 & 5,7 & - & 2
\end{tabular}

Tabelle 8

(s. Abb. 6)

Monatliche Nährstoffgehalte von Mya arenaria in \% des gesamten Trockengewichts während der Untersuchungsperiode 1946.

Juli sehen wir die Korrelation zwischen Glykogen und Ätherextrakt, wie sie von Mytilus bereits im zeitigen Frühjahr bekannt ist. In charakteristischer Weise steigt ebenfalls die Indexkurve im Frühjahr viel markanter an als bei
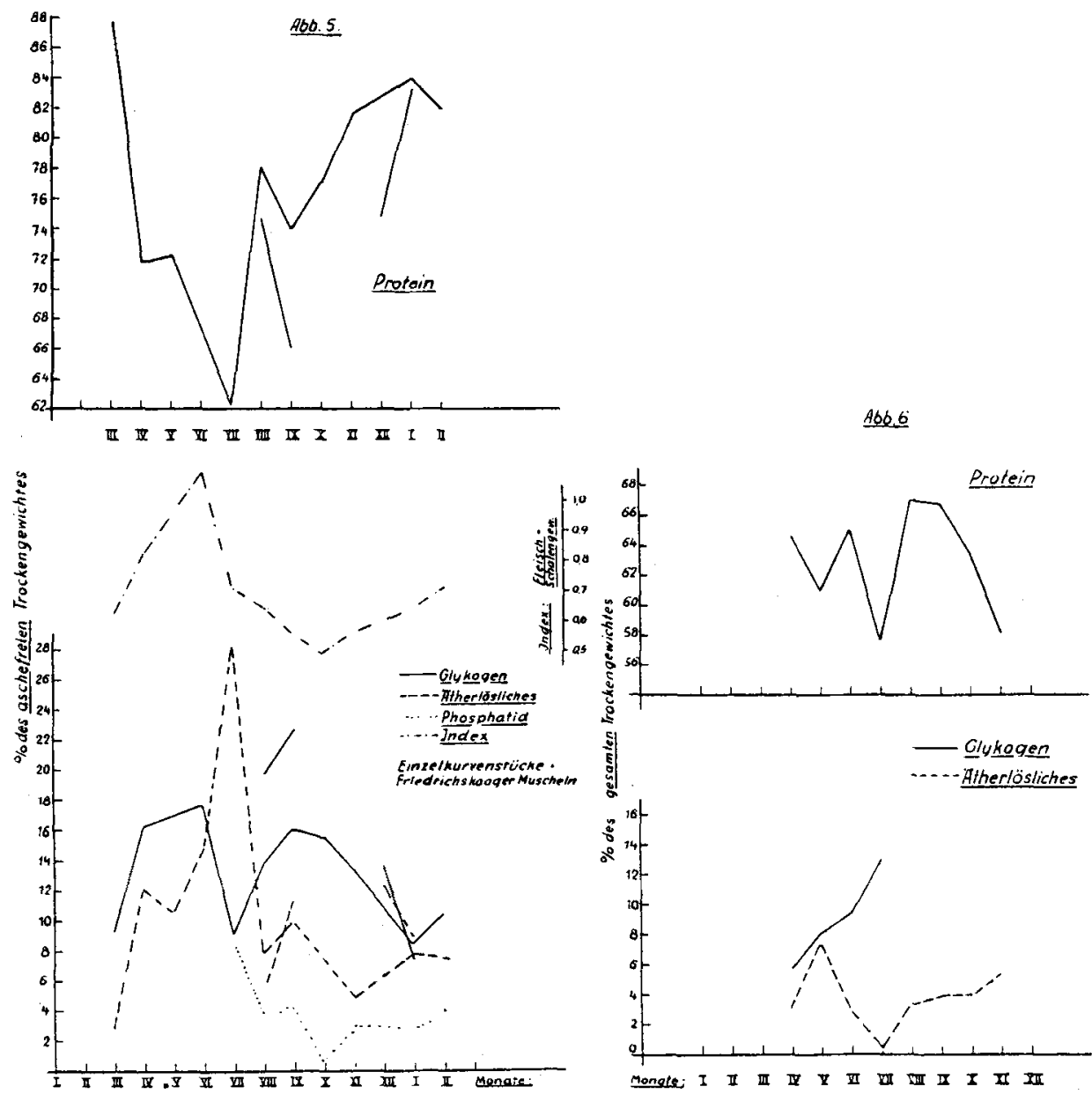

Abb. 5. Monatliche Nährstoffgehalte von Mya arenaria in $\%$ des aschefreien Trockengewidhts während der Untersuchungsperiode $1947 / 48$.

Abb. 6. Monatliche Nährstoffgehalte von $M y a$ arenaria in $\%$ des gesamten Trockengewichts während der Untersuchungsperiode 1946. 
Mytilus, ein Zeichen, daß die Speichertätigkeit im Frühjahr sehr groß sein muß.

Später in der vegetativen Wachstumsphase steigt Glykogen und Protein gleichzeitig auf Kosten des Fettgehaltes an. Die charakteristische GlykogenProteinverschiebung, wie wir sie auch von Mytilus kennen, beginnt sich von September an bemerkbar zu machen.

Die in den Sommer verschobene Laichperiode und das mehr gedrängte Wachstum in der vegetativen Periode charakterisieren den Stoffwechselzyklus von Mya. Das ist auffallend und interessant. Mya ist ein zirkumpolares Tier, dessen südliche Verbreitungszone nicht viel südlicher liegt als es dem Vorkommen an der deutschen Küste entspricht. Mytilus dagegen ist durchaus ein Tier der gemäßigten Zone, geht viel weiter nach Süden, ist z. B. auch im Mittelmeer häufig. Die Lage der Laichzeiten dieser beiden Muschelarten kann nicht herkunftsbedingt sein, sondern muß mit der Eigenart des Stoffwechselzyklus zusammenhängen. Die im tiefen Schlick der Wattenmeere lebende Mya hat im Winter weniger unter dem Absinken der Temperatur zu leiden, während im Sommer die Erwärmung geringer sein dürfte. Im Winter, zu einer Zeit relativer Nahrungsarmut, wird ihre Lebenstätigkeit höher, im Sommer, während einer Zeit relativen Nahrungsreichtums, wird ihre Aktivität niedriger liegen. Beides wirkt der Ansammlung großer Glykogenmengen im Körper entgegen. Hierin ist wohl der Grund zu suchen, daß diese Muschel ihre Laichzeit ins späte Frühjahr und in den Sommer verschieben muß.

$\mathrm{Daß}$ auch ökologische Faktoren bestimmend sein müssen, zeigen die wenigen Werte, die wir von den Friedrichskooger Muscheln gewonnen haben (siehe Tab. 9 u. Abb. 5). Die Kurvenstrecken nehmen sich wie Abschnitte von Gesamtkurven aus, die den Lister Kurven parallel laufen, die aber viel extremere Werte repräsentieren, als die Lister Muscheln sie aufweisen. Die Schwankungen hydrographischer Art werden in dem vom Elbwasser noch stark beherrschten Friedrichskooger Seegebiet größer sein als im Lister Wattenmeer. Eine weitere Beobachtung und Verfolgung dieser hier aufgezeigten Probleme dürfte daher größtes Interesse haben.

\begin{tabular}{cccccc}
\multicolumn{2}{l}{ Monat } & Protein & Fett & Glykogen & Zahl der Tiere \\
\hline VIII & 1947 & 74,3 & 5,8 & 19,9 & 6 \\
IX & 1947 & 66,1 & 11,3 & 22,7 & 6 \\
XII & 1947 & 74,3 & 12,3 & 13,5 & 12 \\
I & 1948 & 83,5 & 8,8 & 7,6 & 7
\end{tabular}

Tabelle 9.

Monatliche Nährstoffgehalte von Mya arenaria in \% des aschefreien Trockengewichts Friedrichskooger Klaffmuscheln.

III. Z us a mmenf assung.

1. Miesmuscheln, (Mytilus edulis), die aus dem Wattenmeer bei List auf Sylt stammten, und Klaffmuscheln (Mya arenaria), ebenfalls aus dem Lister Wattenmeer, sowie kleinere Sendungen von Klaffmuscheln aus den Watten bei Friedrichskoog wurden auf ihren Gehalt an Nährstoffen und zwar auf ihren Gehalt an Protein, ätherlöslicher Substanz, Glykogen und teilweise auch an Phosphatiden untersucht. Die Hauptserien der Muscheln stammten aus den Jahren 1947/48, eine kleinere Serie wurde schon im Jahre 1946 ausgeführt.

2. Die Untersuchungen wurden monatlich ausgeführt. Beide Muschelarten weisen im relativen Gehalt der verschiedenen organischen Substanzen einen charakteristischen Jahreszyklus mit verschiedenen Phasen auf.

Die erste Phase umfaßt die frühjahrsmäßige Laichproduktion und Laichzeit, für Mytilus charakterisiert durch Abnahme relativ hoher Glykogenwerte und Zunahme des Ätherlös- 
lichen, wobei auch Phosphatide einen hohen Prozentsatz ausmachen. Mya geht mit einer geringeren Glykogenreserve in die Laichperiode, ihre Laichzeit ist dementsprechend weiter in den Sommer verschoben.

Die zweite Phase, die nach Absinken des relativen Gehaltes an ätherlöslicher Substanz einsetzt, bezeichnet die sommerliche Periode des vegetativen Wachstums. Sie ist charakterisiert zunächst durch eine Zunahme des relativen Glykogenanteils, später auch durch ein Ansteigen des relativen Gehaltes der Proteine.

Die dritte Phase ist die Periode der winterlichen Ruhe.

3. Ein Vergleich der Ergebnisse der verschiedenen Jahre, sowie der Daten früherer Arbeiten von $D$ IJ_FF und D INIEL mit den von mir ausgeführten zeigt, daß sich die einzelnen Phasen stark verschieben können, was auf den Einfluß geographischer und klimatischhydrographischer Faktoren zurückgeführt wird. Die Unterschiede zwischen Mytilus und Mya wurden mit den eigentümlichen, verschiedenen Lebensweisen der beiden Muschelarten erklärt.

Okologische Faktoren, die den Jahreszyklus ebenfalls beeinflussen, konnten festgestellt, ihre Gesetzmäßigkeiten konnten aber wegen des zu geringen Materials an Friedrichskooger Muscheln noch nicht aufgedeckt werden.

\section{Literatur}

Beythien, A. 1944. Laboratoriumsbestimmungen für den Lebensmittelchemiker, Dresden und Leipzig.

Böhmer, Juckenak und Tillmann, 1936. „Handbuch der Lebensmittelchemie“ 3. Berlin.

B ruce, J. R. 1926. Respiratory Exchange of the Mussel (Mytilus edulis). Biochem. J. 20, 4. Buddenbrock.W. v. 1939. Lehrbuch dex vergleichenden Physiologie 2. Berlin.

Daniel, R. J. 1921. Seasonal Changes in the Chemical Composition of the Mussel (Mytilus edulis). Rep. Lancashire Sea-Fish. Lab. Liverpool 3. Rep. for 1921.

Delf f, Ch. 1912. Beiträge zur Kenntnisnahme der chemischen Zusammensetzung wirbelloser Tiere. Wiss. Meeresunters. Abt. Kiel, N. F. 14.

Ehrenbaum, E. 1884. Untersuchungen über die Struktur und Bildung der Schale der in der Kieler Bucht häufig vorkommenden Muscheln. Z. f. wiss. Zool. 41.

$\mathrm{H}$ a a s, F. 1926. Lamellibranchier, in: Tierwelt der Nord- und Ostsee. Leipzig.

$\mathrm{Hagme}$ ier, A. 1916. Uber die Fortpflanzung der Auster und die fiskalischen Austernbänke. Wiss. Meeresunters. Abt. Helgoland, N. F. 11.

$\mathrm{Hagmeier,A}$, und Kändler, R. 1927. Neuere Untersuchungen im nordfriesischen Wattenmeer und auf den fiskalischen Austernbänken. Wiss. Meeresunters. Abt. Helgoland. N. F. 6.

H a ving a, B. 1929. Krebse und Weichtiere. Handb. d. Seefischerei Nordeuropas, Stuttgart.

Kellog, J. L. 1899. Observations on the Life-History of the Common Clam (Mya arenaria). Bull. U.S. Comm. Fish. 19 for 1899.

- 1909. Conditions governing Existence and Growth of the Soft Clam (Mya arenaria). U.S. Comm. Fish 29. Rep. for 1903.

König, J. 1919. Chemie der menschlichen Nahrungs- und Genußmittel. Nachtrag zu Band $1 \mathrm{~A}$.

Lercbe, Hennert, Halswig und Görtler. 1942. Lehrbuch der tierärztlichen Lebensmittelüberwachung. Hannover.

L inke, O. 1939. Die Biota des Jadebusens. Helgol. wiss. Meeresunters. I.

Meyer, P. F. 1947. Die Sandklaffmuschel, ein wichtiger Eiweißträger. Die Fischwoche, 2.

Mitchell, P. H. 1916. Nutrition of Oysters. The Nature of the socalled Fattening of Oysters. Bull. U.S. Bur. Fish. 35.

$\mathrm{R}$ in ke, H. 1937. Uber die chemische Zusammensetzung einiger Bodentiere der Nord- und Ostsee und ihre Heizwertbestimmungen. Helgol. wiss. Meeresunters. 1.

Russel, E. S. 1923. Report on Seasonal Variation in the Chemical Composition of Oysters. Fish. Invest. Ser. II. 6, London.

$\mathrm{S} \mathrm{t}$ a u ding e r, H. 1942. Glykogenbestimmungen in Organen. Hoppe Seylers Zeitschrift 275.

Wimpenney, R. S. 1938. A Routine Method for the Estimation of Fat in Plankton and its Application to Herring Tissues. Cons. Int. Perm. Explor. Mer, J. du Cons. 13.

Wohlenberg, E. 1937. Die Wattenmeerlebensgemeinschaften im Königshafen von List. Helgol. wiss. Meeresunters. 1.

Yong e, C. M. 1923. The Mechanism of Feeding, Digestion and Assimilation in the Lamellibranch Mya. Brit. J. Exp. Biol. 1. 